\title{
Enhanced Spatial Navigation Skills in Sequence-Space Synesthetes
}

Eline van Petersen ${ }^{1}$, Mareike Altgassen ${ }^{1,2}$, Rob van Lier ${ }^{1}$, Tessa M. van Leeuwen ${ }^{1}$

${ }^{1}$ Radboud University, Donders Institute for Brain, Cognition and Behaviour, Nijmegen, the Netherlands

${ }^{2}$ Department of Psychology, Johannes Gutenberg University of Mainz, Germany

Accepted for publication in Cortex

Correspondence to: Eline van Petersen, email: elinevanpetersen@ gmail.com, Montessorilaan 3, 6525 HR Nijmegen, the Netherlands. 


\begin{abstract}
Individuals with sequence-space synesthesia (SSS) perceive sequences like months, days and numbers in certain spatial arrangements. Several cognitive benefits have been associated with SSS, such as enhanced mental rotation, more vivid visual imagery and an advantage in spatial processing. The current study aimed to further investigate these cognitive benefits, focusing on spatial navigation skills, to explore if their enhanced sensitivity to spatial relations is reflected in enhanced navigational performance. Synesthetes were distinguished from controls by means of a questionnaire, a consistency test and drawings. A virtual Morris Water Maze (MWM) task with two allocentric and two egocentric navigation conditions was used to assess spatial navigation abilities. For the allocentric tasks, participants had to use object cues to find a hidden platform and for the egocentric tasks, they had to use their own position as a reference. Results showed that synesthetes performed significantly better compared to controls on the allocentric and egocentric tasks that reflected real life situations more accurately. However, this significant result was only found for the time taken to find the platform and not for the length of the path that was taken. In exploratory analyses, no significant relations were found between task performance and the specific features of the manifestation of each individual's synesthesia. Our hypothesis that synesthetes with the ability to mentally rotate their spatial arrangements would perform better on the allocentric task was not confirmed. Results add to the growing body of literature concerning the cognitive benefits of SSS and are consistent with the possibility that enhanced spatial navigation skills emerge from generally enhanced visuospatial abilities in SSS.
\end{abstract}

Keywords: sequence-space synesthesia, cognitive benefits, spatial navigation, virtual Morris Water Maze task 


\section{Introduction}

Synesthesia is a phenomenon in which sensory stimulation leads to involuntary additional experiences. Many forms of synesthesia have been reported of which some are common, like perceiving coloured letters (grapheme-colour synesthesia), and some very rare, like tasting words (lexical-gustatory synesthesia). The current study focused on one of the more common forms: sequence-space synesthesia (SSS; Jonas \& Price, 2014). Individuals with SSS perceive months, days of the week, numbers or other sequences in certain spatial arrangements. For instance, months might be seen in a circular form, days in a U-shaped alignment or numbers on a spiralling line (Jonas \& Price, 2014). SSS has previously been associated with several cognitive benefits, some resulting from direct usage of the synesthetic experiences during task performance (e.g., enhanced memory for dates of events; Simner, Mayo, \& Spiller, 2009) and some resulting from a general advantage in a certain cognitive domain (e.g., stronger visual imagery; Havlik Carmichael, \& Simner, 2015; Price, 2009; Rizza \& Price, 2012). The current study aimed to further investigate the cognitive benefits of SSS, focusing on spatial navigation skills, to explore if the enhanced sensitivity of sequence-space synesthetes to spatial relations is reflected in enhanced navigational performance.

\subsection{Characteristics of sequence-space synesthesia}

Some sequence-space synesthetes have a synesthetic percept of only one sequence, whereas others see more than a dozen different sequences (Eagleman, 2009). Visualising numbers and time units, like days, months and years are most common, but all kinds of sequences can elicit visuospatial impressions, even the alphabet, temperatures, or shoe sizes (Eagleman, 2009). The specific sequences that elicit visuospatial impressions comprise just one of the many aspects that can vary among individuals with SSS. For instance, the perceived sequences can take many different shapes of varying complexity (Jonas \& Price, 2014), from simple lines, bent lines, and zigzag lines to circles, squares, and triangles (Eagleman, 2009) and even elaborate three-dimensional landscapes (Brang, Miller, McQuire, Ramachandran, \& Coulson, 2013). Some synesthetes experience the shapes in mental space (associators), others experience the shapes outside of their body (projectors; Jonas \& Price, 2014). For some, the forms are fixed, while others are able to apply spatial transformations, like mentally rotating them and zooming in or out in order to see them from multiple viewpoints (Jonas \& Price, 2014). Some synesthetes additionally experience detailed visual content, such as colour or texture (Jonas \& Price, 2014). Spatial forms might also be seen in two or three dimensions or in first person or third person perspective (Eagleman, 2009). 
For each sequence-space synesthete, the perceived spatial arrangements and characteristics are likely to be unique and can even be different for the different sequences that he or she is able to visualise (Jonas \& Price, 2014). For example, the spatial form for months might be seen from different viewpoints with the passage of time, whereas the form for the alphabet might not involve spatial transformations because it does not change over time (Jonas \& Price, 2014). Moreover, the spatial arrangements often involve personal importance, like distortions of date lines to mark personally significant events (Price \& Pearson, 2013) or important months occupying more space than others (Brang et al., 2013). Despite these various manifestations of SSS, it is common for all sequence-space synesthetes that seeing, hearing or thinking about particular sequences, as a whole or in parts, involuntarily elicits additional visuospatial experiences that are consistent over time (Cohen Kadosh, Gertner, \& Terhune, 2012; Price \& Pearson, 2013).

\subsection{Cognitive benefits and costs of sequence-space synesthesia}

Previously, it has been shown that visuospatial associations in SSS are beneficial for several cognitive tasks. Sequence-space synesthetes with time forms (e.g., months or years) outperform non-synesthetes in tests that assess recall of dates of public events and content of events in their own life. Synesthetes subsequently reported that events were retrieved from spatial locations within their visuospatial forms (Simner et al., 2009). It seems that the additional experiences in SSS lead to richer encoding and retrieval opportunities during memory tasks (Rothen, Meier, \& Ward, 2012). Moreover, sequence-space synesthetes seem to be better in acquiring the skill of calendar calculation (i.e., the ability to give the correct day of the week for any given date; Hughes, Gruffydd, Simner, \& Ward, 2019). These advantages seem to be directly related to the SSS experiences themselves, i.e., synesthetes were using their synesthetic experience when performing the task. Several other studies have also shown general advantages that come along with SSS. Sequence-space synesthetes report a richer general imagery experience than controls (Havlik et al., 2015; Price, 2009; Rizza \& Price, 2012), which in turn is suggested to support performance on mental rotation tasks on which synesthetes demonstrate enhanced performance as well (Brang et al., 2013; Havlik et al., 2015). Sequencespace synesthetes were also found to have an advantage in spatial processing ability in the domain of their synesthetic concurrent experience (i.e., space). This was demonstrated with a task comprising spatial stimuli (i.e., several circles differing in size), where participants were required to make judgements about the overlapping order (i.e., leftmost circle on the bottom and rightmost on the top, or the other way around; Hale, Thompson, Morgan, Cappelletti, \& 
Cohen Kadosh, 2014). This link to increased spatial processing has also been demonstrated by means of a questionnaire about cognitive styles (Mealor, Simner, Rothen, Carmichael, \& Ward, 2016). Moreover, the study by Hale et al. (2014) suggested a general difference in working memory between sequence-space synesthetes and non-synesthetes since synesthetes demonstrated higher visuospatial working memory accuracy compared to controls.

However, the consistent and involuntary synesthetic experiences have some costs as well. Sequence-space synesthetes respond slower on target detection tasks when the spatial relationship between items of presented sequences, like months (Smilek, Callejas, Dixon, \& Merikle, 2007) and numbers (Gertner, Henik, \& Cohen Kadosh, 2009), is incongruent with their own visuospatial percept of those sequences. This suggests that SSS "impairs the ability to represent items of sequences in a flexible manner according to task demands" (Gertner et al., 2009, p. 366). Furthermore, it has been shown that synesthetes with number forms are slower in doing simple calculations (Ward, Sagiv, \& Butterworth, 2009). This could perhaps be the case because they are relying on their visuospatial forms when solving these arithmetic problems instead of using rote retrieval (i.e., a memorization strategy based on repetition), which is more optimal in this case (Hale et al., 2014).

\subsection{Sequence-space synesthesia and spatial navigation}

In several studies investigating synesthesia and its benefits, synesthetes have shown improved performance in the domains of their synesthetic concurrent experiences. For instance, grapheme-colour synesthetes are better at colour discrimination and mirror-touch synesthetes are better at tactile discrimination due to enhanced sensitivity in these domains (Yaro \& Ward, 2007; Banissy, Walsh, \& Ward, 2009). These findings imply that synesthesia is accompanied by a general processing benefit in the domain of the synesthetic concurrent experience, outside of the synesthetic experience itself (Banissy et al., 2009). As mentioned before, sequence-space synesthetes have already shown improved processing ability in the domain of their synesthetic concurrent experience (i.e., space; Hale et al., 2014). Sequence-space synesthetes may thus have a general spatial advantage because of their enhanced sensitivity to spatial relations, which may enhance spatial navigation performance. In addition, the previously reported cognitive benefits of SSS (e.g., superior imagery, memory and mental rotation performance), linked to spatial navigation (e.g., Behrmann \& Shomstein, 2009; Harris, Wiener, \& Wolbers, 2012), might aid navigational performance. Evidence from patient studies support the role of memory and imagery in spatial navigation. Patients suffering from representational neglect are affected in memory and spatial imagery performance (Chersi \& Burgess, 2015) and it has been shown that 
they experience deficits in navigation when they have to re-orient themselves (Guariglia, Piccardi, Iaria, Nico, \& Pizzamiglio, 2005). This suggests that when memory and imagery performance are affected, spatial navigation abilities are likely to be affected. Similarly, the improved memory and imagery performance of sequence-space synesthetes together with their possible general spatial advantage due to enhanced sensitivity to spatial relations, may enhance their spatial navigation abilities. To date, this has not yet been explored, which is surprising given that spatial navigation is an important and evolutionary relevant skill to have.

Guariglia et al. (2005) used a human version of the Morris Water Maze (MWM) task in real space to test spatial navigation skills in patients with mental representation disorders. The original MWM task was developed for rats and required them to find a platform by using various cues while moving around in a pool (Morris, 1981). In the human version of the task used by Guariglia et al. (2005), participants had to explore a room and find a target location. This task only required target place learning from different starting positions. The current study used a computerized version of the MWM task similar to the one used by Ring, Gaigg, Altgassen, Barr and Bowler (2018) that was adapted from Feigenbaum and Morris (2004). In this version of the task, a virtual pool was presented on a touchscreen and participants were required to find a hidden platform by moving over the screen. Over trials they had to work out and learn the shortest possible path from the starting point to the platform. For some conditions, they had to make use of object cues (allocentric) and for other conditions they had to use their own position as a reference (egocentric) in order to find the platform.

Differentiating between allocentric and egocentric conditions is relevant because normally when navigating in an environment both allocentric and egocentric strategies can be used. When using an allocentric strategy - also called place strategy or spatial memory strategy - one uses cognitive maps (i.e., mental representations of an environment) by thinking about landmarks and their positions relative to each other (e.g., Di Tore, Corona, \& Sibilio, 2014; Iaria, Petrides, Dagher, Pike, \& Bohobot, 2003; Konishi \& Bohobot, 2013). When using an egocentric strategy - also called response or route strategy - one navigates by following a learned sequence of self-movements, such as a series of left and right turns at precise decision points from a given starting position (e.g., turn right after the park; e.g., Bohobot, Lerch, Thorndycraft, Iaria, \& Zijdenbos, 2007; Chersi \& Burgess, 2015; Di Tore et al., 2014; Konishi $\&$ Bohobot, 2013). The MWM task involved two allocentric and two egocentric conditions of which the first conditions (i.e., Allocentric 1 and Egocentric 1) were more similar to navigation in daily environments. 
Previous studies have demonstrated that the virtual version of the MWM task is sensitive to detect differences in spatial navigation between certain groups. Ring et al. (2018) demonstrated that individuals with autism spectrum disorder (ASD) have difficulties in allocentric navigation, particularly when the task required them to change position while the platform and objects kept the same locations (i.e., the Allocentric 1 condition). Feigenbaum and Morris (2004) also found impairment on this allocentric navigation task in patients who had undergone right temporal lobectomy (RTL). Using this virtual MWM task, the current study set out to investigate whether such differences in navigational performance exist between sequence-space synesthetes and non-synesthetes.

Besides comparing spatial navigation skills between the two groups, individual differences among sequence-space synesthetes were examined when assessing spatial navigation abilities in order to see whether some specific synesthetic features were associated with performance. Specifically, the ability to mentally rotate spatial forms was expected to enhance performance in at least the allocentric condition, in which the display had to be mentally rotated in order to find the platform. Further influences of synesthetic features on navigational performance were explored as well. Individual differences among sequence-space synesthetes have previously been shown to influence performance on visuospatial tasks. For example, synesthetes who are able to project forms into space (i.e., projector synesthetes) were shown to perform best on mental rotation tasks (Havlik et al., 2015).

The current study aimed to further investigate the cognitive benefits of SSS. Previously, synesthetes have shown improved performance in domains of their synesthetic concurrent experiences, even outside of the synesthetic experience itself. For example, synesthetes experiencing colour demonstrate enhanced colour processing and synesthetes experiencing touch demonstrate enhanced tactile processing (Yaro \& Ward, 2007; Banissy et al., 2009). Consistent findings may be expected for sequence-space synesthetes for whom the domain of the synesthetic concurrent experience is space. Sequence-space synesthetes may show a general spatial advantage because of enhanced sensitivity to spatial relations, which may enhance their navigational performance. Therefore, this study set out to investigate whether sequence-space synesthetes have enhanced spatial navigation skills. We expect synesthetes to outperform nonsynesthetes on a spatial navigation task, and additionally, we expect synesthetes with the ability to mentally manipulate their spatial arrangements (i.e., applying mental rotations) to outperform synesthetes without this ability on the allocentric navigation task involving mental rotation. Extending our knowledge of the cognitive capacities that come along with SSS will contribute 
to the growing body of literature concerning the cognitive benefits of SSS and will inform us on how allocentric and egocentric navigation is normally accomplished.

\section{Methods}

We report how we determined our sample size, all data exclusions, all inclusion/exclusion criteria, whether inclusion/exclusion criteria were established prior to data analysis, all manipulations, and all measures in the study.

\subsection{Participants}

Participants with SSS were recruited to the study through poster advertisements and via the Radboud research participation system, clearly advertising it as an SSS study to attract synesthetes. Age- and sex-matched control participants were recruited via the research participation system as well, advertising it as a study involving several cognitive tasks. Over one hundred individuals completed the initial online screening questionnaire about synesthetic experiences. After evaluating the responses, 23 potential synesthetes and 22 controls were invited to take part in the study. Synesthetes were chosen based upon the most promising answers (i.e., the number of sequences they reported to visualise and the richest description of experiences) and controls were chosen to match these synesthetes (N.B., based on age, gender and education level). Further inclusion criteria comprised accurate sight, no health issues, and no use of medication or drugs that could potentially influence visual perception. After the tasks in the lab, one potential synesthete was excluded from the synesthete group (due to insufficient responses on the consistency task and a drawing without typical synesthetic characteristics) and one potential control participant appeared to be in fact a synesthete and was accordingly allocated to the synesthete group. The groups that were taken into account in analysis comprised 23 individuals with SSS (20 women, $M_{\text {age }}=23.22$ years, age range 18-25 years with three exceptions (34, 38 and 44 years, included in the mean age)) and 21 controls (19 women, $M_{\text {age }}$ $=21.57$ years, age range 18-25 years). An independent samples t-test indicated no significant age difference between groups $(t(26)=1.15, p=.263)$. Because of unequal variances between groups (Levene's test was significant), the degrees of freedom were adjusted accordingly. Of those included in the synesthete group, 13 reported having spatial forms for numbers, 21 for days and 23 for months. All participants were educated at university level. Informed consent was obtained before filling out the online screening questionnaire and again in the lab before taking part in the experiment. Participation was voluntary and compensated with 15 euros or 
1.5 credit points. The study was approved by the Ethics Committee of the Faculty of Social Sciences (ECSS) at Radboud University Nijmegen.

A power calculation (Faul, Erdfelder, Lang, \& Buchner, 2007) indicates that with 44 participants in the analyses and a power of 0.80 , we should be able to detect between-within interaction effects in the main ANOVA analyses of the MWM task (within-subject factors condition (2 levels) and trial (16 levels), between-subject factor group (2 levels)) with a small effect size $\left(\eta_{\mathrm{p}}^{2} \approx .009\right)$, and between-groups effects with slightly higher but still intermediate effect size $\left(\eta_{\mathrm{p}}^{2} \approx .088\right)$.

\subsection{Tasks and procedure}

\subsubsection{General procedure}

Before participating in the study, all participants filled out an online self-report questionnaire about synesthetic experiences. In the lab, by means of a consistency test, drawings, and additional questions, sequence-space synesthetes were distinguished from control participants. Participants were asked to select locations on a computer screen for numbers, days, and months, yielding a consistency score of the placement of items, and to draw their visuospatial experiences (synesthetes) or intuitive representations (controls) of those sequences on paper. Then participants performed a virtual MWM task to assess spatial navigation skills. During allocentric and egocentric navigation tasks, participants were asked to find a hidden platform by moving over a touchscreen. For the allocentric tasks, they had to use object cues to find the platform and for the egocentric tasks, they had to use their own position as a reference to find the platform. Performance on these different tasks was assessed between groups and individual differences in the manifestation of SSS were taken into account in further exploratory analyses. We now describe each task in detail.

\subsubsection{Screening questionnaire}

An online SSS self-report questionnaire was developed in LimeSurvey and used as a screening tool to find participants for our study. The questions were based on descriptions of SSS in the literature and comprised some general screening questions (e.g., "Is the synesthetic experience automatically elicited when thinking of this sequence?") and some detailed questions about the perceived spatial forms (e.g., "Do you see this arrangement from a fixed perspective or are you able to rotate the form and adopt multiple viewpoints?"). When participants reported to have SSS for numbers, days and/or months, detailed questions followed about the spatial forms of each of those sequences separately. These questions covered all the characteristics of SSS as mentioned in the theoretical background. The complete questionnaire 
can be found in the Supplementary Materials (translated from Dutch to English). Filling out the questionnaire took about 10 to 20 minutes. Participants who reported having SSS received an invitation to participate in the study at Radboud University. Control participants filled out the questionnaire as well. They could just simply answer the first question ("Do you think you have sequence-space synesthesia?") with "no" and the specific questions about SSS did not appear. Before filling out the questionnaire, all participants were provided with a description of SSS and some examples of visuospatial forms to familiarize all of them with SSS prior to completing the questionnaire. This description and these example forms can be found in the Supplementary Materials.

\subsubsection{Consistency test}

Because the screening questionnaire was based on self-report, participants' subjective reports of SSS were verified in the lab. Participants were asked again about the details of their spatial forms and performed a consistency test. The consistency test (Rothen, Jünemann, Mealor, Burckhardt, \& Ward, 2016), written in E-prime 2.0, was obtained from Rothen et al. (2016) and adapted to the current study. Numbers $0-9,50$ and $100(N=12)$, days $(N=7)$ and months $(N=12)$ were centrally presented on a white background with font style Courier New and font size 18 in bold black. These stimuli were presented on a 24" BenQ screen with display resolution set to 1920x 1080, controlled by a Dell computer running Windows 7 .

Participants were comfortably seated in front of the screen at normal viewing distance. Stimuli were presented one by one in random order and participants had to select a location for each stimulus by clicking on the screen. SSS participants were instructed to imagine the screen as the space in which they experienced the spatial arrangements of the sequences and choose locations that best fit their synesthetic experience. When a presented stimulus did not induce a synesthetic experience, they could press the space bar and the next stimulus appeared. There were five practice trials to get familiar with the task. Control participants were asked to find an intuitive location for each stimulus. They were instructed to try to choose the same location every time the stimulus reappeared, but they were not allowed to choose the same location for every single stimulus. Control participants did not have the opportunity to press the space bar. Afterwards they were asked if they had used a certain strategy for placing the different stimuli.

Each stimulus was presented for $1 \mathrm{~s}$ in the centre of the screen, then a cross appeared and participants could choose a location for the stimulus. All stimuli were presented three times resulting in a total of $93(=31 \times 3)$ trials. Completing this task took about 15 minutes. The three chosen locations for each item formed a triangular area and we used the mean surface of all 
these areas together as the consistency score of each participant, according to the procedure described by Rothen et al. (2016).

\subsubsection{Drawings}

After the consistency test, SSS participants were asked to draw their spatial forms on a piece of paper and control participants were asked to draw a representation of numbers, days and months. Sequence-space synesthetes could use coloured pencils if they experienced additional colours with their spatial forms. It was verified whether control participants really associated those locations with the sequences or whether they just chose the same locations as remembered from the consistency test. These drawings were used as a control measure for the consistency test because control participants could achieve high consistency scores as well when adopting a certain strategy, for example placing items of a particular sequence in a straight line from left to right. Besides giving more confidence in distinguishing real sequence-space synesthetes from controls, these drawings were for some SSS participants an easier method to express their visuospatial experiences. This was especially relevant for those who perceived sequences with high visual content like colours because the consistency test was only a purely spatially-based estimate (Jonas \& Price, 2014). This task took an additional 5 to 15 minutes, depending on the complexity of the drawings.

\subsubsection{Morris Water Maze task}

Finally, after the consistency test and drawings, participants performed a computerized version of the Morris Water Maze (MWM) task (Ring et al., 2018) to assess spatial navigation abilities. This task was written in Microsoft Visual Basic 6 and presented on a 19" ELO touchscreen with display resolution set to $1280 \times 1024$, controlled by a Dell computer running Windows XP. The screen was placed on a square table at comfortable height such that participants could easily reach the screen while standing. The table was surrounded by black curtains hanging from the ceiling to the ground and formed a $9 \mathrm{~m}^{2}$ separate area inside the room (Fig. 1). This prevented participants from using environmental cues, like doors and features on the wall, to guide navigation. There was enough space around the table such that participants could easily walk around the screen as instructed during the task. Lights were turned off to further reduce the influence of cues in the room. 


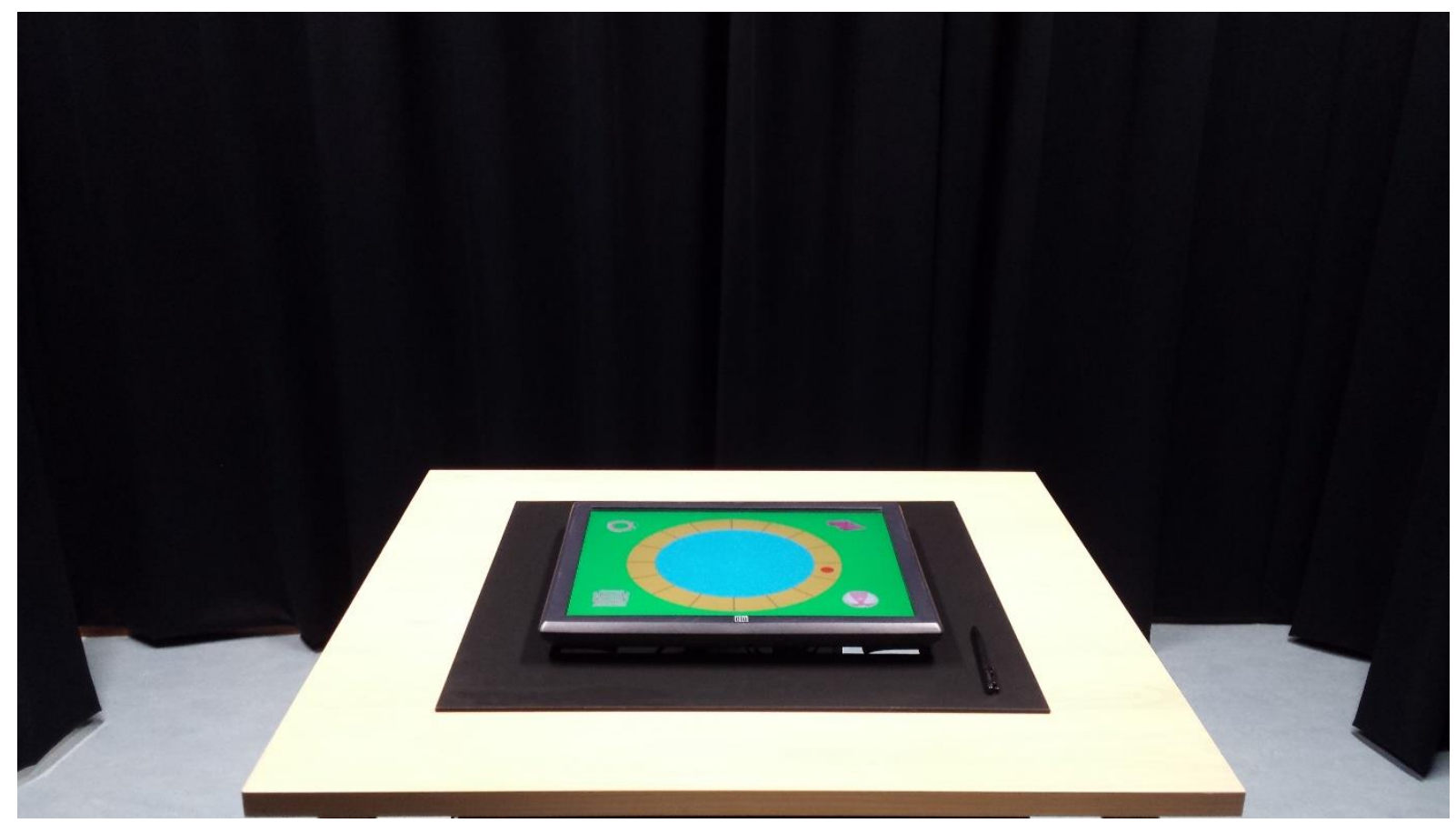

Fig. 1. Experimental set-up.

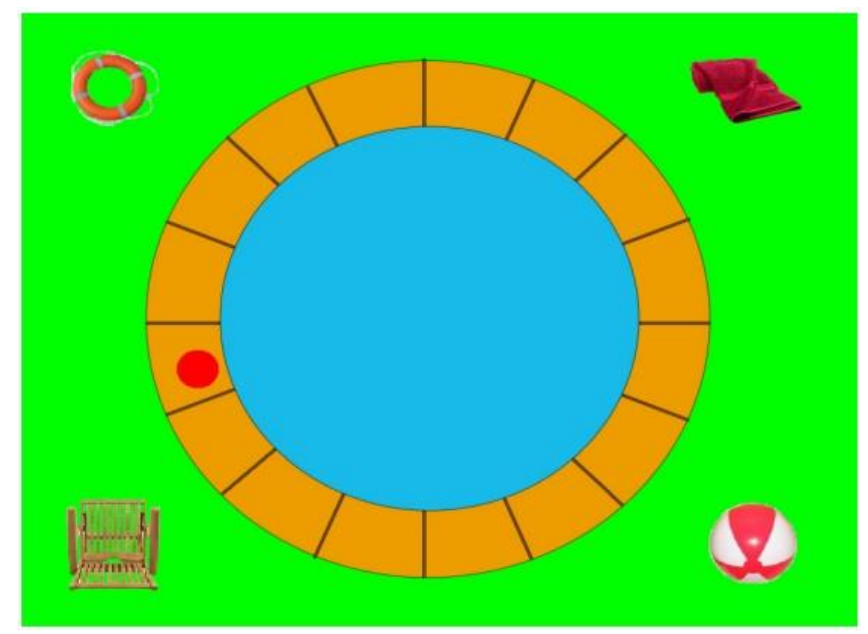

Fig. 2. Example of display.

On every trial, a virtual swimming pool environment was presented on the touchscreen. The display consisted of a blue circular area surrounded by an orange wall representing water and the border of the pool, respectively. The green area outside the pool represented grass. There were four object cues (life ring, towel, chair, beach ball) around the pool, one in each corner of the screen (Fig. 2). Participants were instructed to find a hidden platform in the pool by moving over the touchscreen. They always had to start moving over the screen from the red dot that was presented in a fixed randomized order at the orange border. While searching for the platform, they used a touchscreen sensitive pen because this pen moved more easily over 
the screen than their finger and was therefore more accurate in registering the path that was taken. Participants were not allowed to lift the pen from the screen while searching for the platform and they were not allowed to cross the border of the pool. The platform, presented as a brown box, appeared once they passed the right location.

Participants were asked to work out and learn the shortest possible path from the starting point (i.e., red dot) to the hidden platform over several trials. There were three practice trials to get familiar with the task and to learn how to move properly over the screen. After these practice trials, participants had to perform five tasks, each consisting of 16 trials. The first task was always a place learning block and then two allocentric and two egocentric navigation blocks followed. These allocentric and egocentric blocks could be presented in any possible order, making a total of 16 different task orders. It was made sure that every order was performed by at least one synesthete and one control participant. For the allocentric tasks, participants had to use the object cues to find the platform and for the egocentric tasks, they had to use their own position as a reference. Importantly, participants had to figure out 'the rule' for finding the platform themselves.

\subsubsection{Place learning}

Place learning was used as a control condition to ensure that participants were able to perform the task properly and to check whether they showed learning over trials. There were no systematic manipulations during this condition. The objects, platform and participant kept the same positions (Fig. 3A).

\subsubsection{Allocentric conditions}

The two allocentric conditions were used to measure the strength of allocentric processing. In the first allocentric condition (Allocentric 1), the objects and platform stayed in the same location, but the participant changed position. The participant had to move to another side of the screen after every trial. This happened in a fixed randomized order. This condition was the original allocentric condition as developed by Feigenbaum and Morris (2004; Fig. 3B). In the second allocentric condition (Allocentric 2), the participant stayed in the same position, but the objects and platform changed in a fixed randomized order. The platform moved along with the objects, so they kept the same positions relative to each other. Participants did not see the platform and objects rotating, they only saw the new rotated order. This condition was added to the task by Ring et al. (2018; Fig. 3C).

\subsubsection{Egocentric conditions}

The two egocentric conditions were used to measure the strength of egocentric processing. In the first egocentric condition (Egocentric 1), the platform and participant stayed 
in the same location, but the objects rotated in a fixed randomized order. Participants did not see the objects rotating, they only saw the new rotated order. This condition was the original egocentric condition as developed by Feigenbaum and Morris (2004; Fig. 3D). In the second egocentric condition (Egocentric 2), the objects stayed in the same location, but now the platform and participant changed position in a fixed randomized order. The platform moved along with the participant, so platform and participant kept the same positions relative to each other. This condition was added to the task by Ring et al. (2018; Fig. 3E).

For each trial participants had 60 seconds to find the platform. When a participant could not find the platform within these 60 seconds, a time out message appeared together with the platform. Every trial was followed by a distractor task. Participants had to 'pop' ten blue bubbles that appeared one by one at random locations on a black screen. After this distractor task, a black screen appeared with a yellow dot at one of the four sides of the screen, indicating on which side the participant had to stand for the upcoming trial.

After performing this virtual MWM task, participants were asked about their strategy for solving the task in order to control for the use of allocentric strategies in the allocentric conditions and egocentric strategies in the egocentric conditions. Additionally, they were asked about their navigational strategies in daily life. Performing the MWM task took about 30 minutes. 

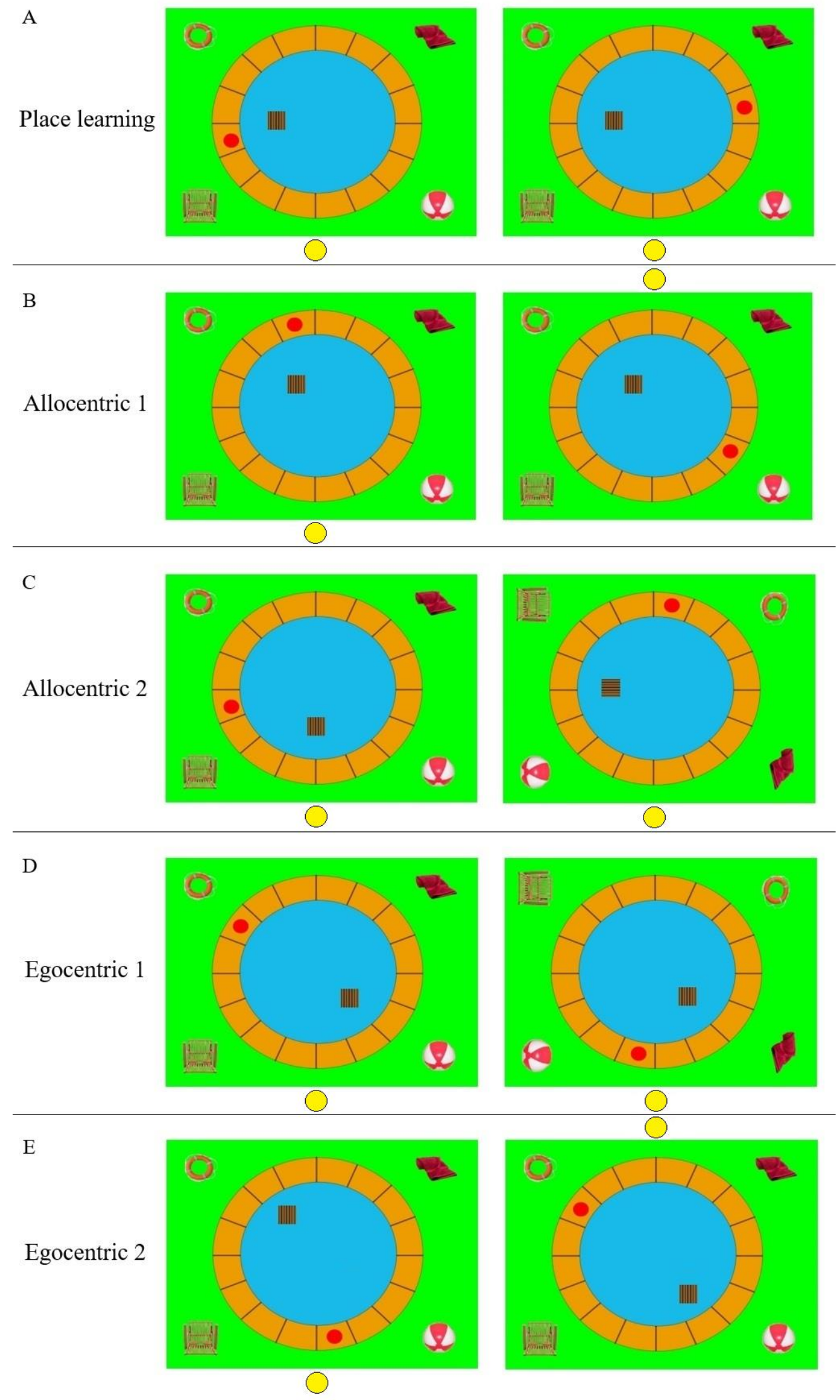

Fig. 3. Two example trials of each condition (A-E). The yellow dot indicates the standing position of the participant relative to the screen, which could be on any of the four sides of the screen. The red dot indicates the starting position for moving over the screen. The platform was not visible for participants during the task, only after they passed the right location. 


\section{Results}

Data of the consistency test were analysed using t-tests and data of the MWM task using repeated measures analyses of variance (ANOVAs). If the sphericity assumption was violated, Greenhouse-Geisser correction (GG) was applied. The significance level for all analyses was set at $\alpha=.05$.

\subsection{Consistency test}

The three chosen locations for each item of the consistency test formed a triangular area and the mean surface in pixels across all these areas was our measure of consistency. The lower the score, the higher a participant's consistency of placing the items. Given that sequence-space synesthetes always have the same spatial association for certain sequences, they were expected to consistently choose the same locations for the presented items. Controls do not have these spatial associations, therefore they were expected to choose these locations less consistently.

When taking the consistency scores for numbers, months and days together, an independent-samples t-test indicated that sequence-space synesthetes performed significantly more consistently than controls $(t(22)=-2.18, p=.040, \Delta=.49,95 \%$ CI $[421.28,7892.72])$. Also for numbers and months separately - but not for days - sequence-space synesthetes performed significantly more consistently: for numbers $(t(21)=-2.09, p=.049, \Delta=.46,95 \%$ CI $[240.56,7339.44])$ and for months $(t(21)=-2.10, p=.048, \Delta=.46,95 \%$ CI [360.30, 10307.70]). Because of unequal variances between groups (Levene's test was significant), the degrees of freedom were adjusted, and Glass's delta was used for determining the effect size. The descriptive statistics of both groups are summarized in Table 1 and the spreading of the individual consistency scores for numbers, months and days together are shown in Figure 4. Despite the significant difference in consistency between groups, this figure clearly shows a lot of overlap in the consistency scores between synesthetes and controls. A few examples of generated figures of performance of both sequence-space synesthetes and control participants are presented in the Supplementary Materials (Fig. S8 and S9). A substantial amount of control participants obtained high consistency scores by using certain strategies for placing the items. Therefore we eventually did not use a synesthesia cut-off score as in Rothen et al. (2016) to classify sequence-space synesthetes and controls. Using only a cut-off score would have led to incorrect classification of participants. 


\section{Table 1.}

Descriptive statistics of the consistency scores in number of pixels of both groups for numbers, months and days together and separately.

\begin{tabular}{lccccc} 
& \multicolumn{2}{c}{ SSS } & \multicolumn{2}{c}{ Con } \\
& $\boldsymbol{M}$ & $\boldsymbol{S D}$ & $\boldsymbol{M}$ & $\boldsymbol{S D}$ & $\boldsymbol{N}$ SSS/Con \\
\hline Total & 2320 & 2125 & 6477 & 8495 & $23 / 21$ \\
Numbers & 1335 & 1119 & 5125 & 8176 & $13 / 21$ \\
Months & 2317 & 1985 & 7651 & 11473 & $23 / 21$ \\
Days & 2456 & 4066 & 5969 & 12417 & $21 / 21$
\end{tabular}

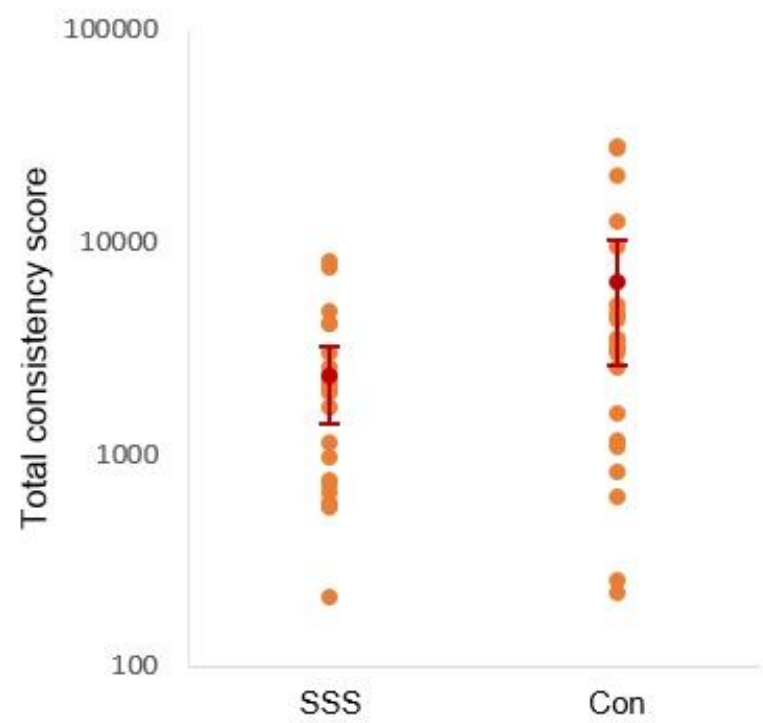

Fig. 4. Spreading of the individual consistency scores of both groups for numbers, months and days together. The red dot indicates the mean and error bars represent the $95 \%$ confidence interval. The scale of the $\mathrm{y}$-axis is logarithmic.

\subsection{Drawings}

Instead of the consistency test, drawings and participants' descriptions of their synesthetic experiences - both from the questionnaire and questions in the lab - gave more confidence in correctly distinguishing sequence-space synesthetes from controls. Drawings were compared between the groups and in addition to the drawings, synesthetes were asked how they perceived their visuospatial forms (e.g., projection vs. association, fixed perspective vs. multiple viewpoints). Controls were asked whether they always perceived the sequences like their drawings or whether the drawn locations were intuitive and not automatically elicited when thinking of those sequences.

A comparison of drawings revealed some striking differences between sequence-space synesthetes and controls. Synesthetes showed a tendency to connect items of a sequence within 
a form (e.g., months connected as blocks in a circle), whereas controls just wrote the items down in isolation (e.g., months scattered on locations where they could remember them). For months, around $70 \%$ of the synesthetes connected items within a form, for days around $67 \%$ and for numbers around $54 \%$. None of the control participants did this. In terms of complexity, drawings made by synesthetes were characterized by more elaborate and complex forms compared to those of controls. For months, around $65 \%$ of the synesthetes drew a circle, oval, square or similar closed form and for days around $43 \%$. For numbers, around $62 \%$ of the synesthetes drew a line with bends, corners or zigzags. None of the control participants did this. Most of them arranged the items of a sequence in rows or columns. For months, around 62\% of the control participants did this, for days around $71 \%$ and for numbers around $67 \%$. A detailed overview of the characteristics of each sequence for both groups can be found in the Supplementary Materials (Table S7 and S8) as well as a few examples of drawings made by sequence-space synesthetes and control participants (Fig. S10 and S11).

In addition, a complexity score was created for synesthetes based on the drawings and descriptions of their forms. For each sequence, ten complexity features were chosen, and one point was given for each feature that was present. The total score was divided by the number of sequences the synesthete perceived (i.e., one, two or three), so the score could range between zero and ten points. This score was used as a covariate in the analyses of the MWM task. An overview of the complexity features is presented in the Supplementary Materials (Table S9).

\subsection{Morris Water Maze task}

For the MWM task, four dependent measures were used to assess performance: (1) the length of the path taken to find the platform (Path Length), (2) the time needed to find the platform (Time to Target), (3) the percentage of time spent in the quadrant containing the platform (Percentage of Time in Target Quadrant), and (4) the angle of the path taken heading towards the platform after the first movement on the screen (Path Angle). We focused in our analyses on Time to Target and Path Length since these variables captured performance most directly and were straightforward to interpret. Moreover, these variables showed a clear learning effect over trials in contrast to the other two measures (Fig. S1 in the Supplementary Materials). For more details on why these latter measures were not included in the main analyses, see General Discussion. The results of Percentage of Time in Target Quadrant and Path Angle are presented in the Supplementary Materials (Table S4 and S5 and Fig. S3 and S4).

Path Length was calculated as the difference between the shortest possible path and the actual path that was taken in order to enable comparison between trials. This measure was then 
transformed from pixel into mm. Time to Target was measured in milliseconds. Because of a very high variation in the data, both Path Length and Time to Target were square root transformed. Due to this transformation, the variation became less extreme for trials with long search times and path lengths (i.e., the first few trials). As in Ring et al. (2018), the analyses were done for the Allocentric 1 and Egocentric 1 conditions and for the Allocentric 2 and Egocentric 2 conditions. These analyses were done separately for conditions 1 and 2, because the first conditions were the original conditions of the MWM task developed by Feigenbaum and Morris (2004) - and reflected real life situations more accurately (see General Discussion) - and the second conditions were the added conditions developed by Ring et al. (2018).

The complete tables with descriptive and inferential statistics for Time to Target and Path Length are presented in the Supplementary Materials (Table S2 and S3). For Time to Target, not more than $2 \%$ of the trials per condition were outliers and for Path Length this was not more than 3\% (see Supplementary Materials for how outliers are dealt with).

\subsubsection{Place learning}

Data were analysed using a repeated measures ANOVA with the between-subjects factor group (SSS, controls) and the within-subjects factor trial (16 trials). For performance measured by Time to Target, a significant main effect was found for trial, $F(7.26,304.98)=$ 20.21, $p<.0001, \eta_{\mathrm{p}}{ }^{2}=.33$, GG, meaning that the time needed to find the platform decreased over trials. There was no significant main effect for group or a significant group $\mathrm{x}$ trial interaction, which indicates similar learning over trials for both groups. Similar results were found for performance measured by Path Length (Table S1 and Fig. S1 in the Supplementary Materials).

\subsubsection{Allocentric 1 and Egocentric 1}

Data were analysed using a repeated measures ANOVA with the between-subjects factor group (SSS, controls) and the within-subjects factors trial (16 trials) and condition (allocentric, egocentric). For performance measured by Time to Target, significant main effects were found for trial, $F(6.40,268.77)=45.33, p<.0001, \eta_{\mathrm{p}}{ }^{2}=.52$, GG, and for condition, $F(1$, $42)=6.44, p=.015, \eta_{\mathrm{p}}{ }^{2}=.13$, showing that the time needed to find the platform decreased over trials and that it took more time to find the platform in the allocentric condition $(M=47.47, S D$ $=15.67)$ compared to the egocentric condition $(M=41.71, S D=14.02)$ for most trials, suggesting that the allocentric condition was more difficult. Importantly, a significant main effect of group, $F(1,42)=4.46, p=.041, \eta_{\mathrm{p}}{ }^{2}=.10,95 \%$ CI $[0.62,15.12]$, indicated that sequence-space synesthetes performed better at both conditions $(M=40.83, S D=13.17$, range $=22.68-73.74)$ compared to controls $(M=48.70, S D=11.36$, range $=33.26-71.14)$. There was 
no significant group x condition interaction. Figures $5 \mathrm{~A}$ and $\mathrm{B}$ show performance over trials for both groups for the Allocentric 1 and the Egocentric 1 conditions, respectively. A similar trend was found for performance measured by Path Length, but for this dependent variable the main effect of group did not reach significance $\left(F(1,42)=2.71, p=.107, \eta_{\mathrm{p}}{ }^{2}=.06,95 \%\right.$ CI [2.47, 24.35]; Table S2 and Fig. S2 in the Supplementary Materials).

\subsubsection{Allocentric 2 and Egocentric 2}

Data were analysed using a repeated measures ANOVA with the between-subjects factor group (SSS, controls) and the within-subjects factors trial (16 trials) and condition (allocentric, egocentric). For performance measured by Time to Target, significant main effects were found for trial, $F(6.07,255.11)=26.37, p<.0001, \eta_{\mathrm{p}}{ }^{2}=.39$, GG, and for condition, $F(1$, $42)=4.17, p=.048, \eta_{\mathrm{p}}{ }^{2}=.09$, showing that the time needed to find the platform decreased over trials and that it took more time to find the platform in the allocentric condition $(M=51.00, S D$ $=16.12)$ compared to the egocentric condition $(M=46.94, S D=18.35)$ for most trials, suggesting that the allocentric condition was more difficult. There was a significant trial $\mathrm{x}$ condition interaction as well, $F(8.17,343.08)=3.54, p=.001, \eta_{\mathrm{p}}{ }^{2}=.08$, GG, indicating that the time needed to find the platform decreased more over trials for the allocentric condition compared to the egocentric condition. There was no significant main effect of group, $F(1,42)$ $=.046$, n.s., $95 \%$ CI $[-8.28,10.38]$ or a significant group $\mathrm{x}$ condition interaction, meaning that sequence-space synesthetes and controls showed similar performance in both conditions. Figures 5C and D show performance over trials for both groups for the Allocentric 2 and the Egocentric 2 conditions, respectively. Similar results were found for performance measured by Path Length, except for a significant main effect of condition (Table S2 and Fig. S2 in the Supplementary Materials).

\subsubsection{Control measures}

There were several measures to control for correct performance. It was counted how often participants left the pool area, how often they lifted the pen from the screen, when they were timed out (i.e., when they could not find the platform within 60 seconds), and how much time they needed to complete the distractor task. Control measures were analysed using repeated measures ANOVAs. There were no significant differences between groups for any of these measures, meaning that any variations in the ability to correctly perform the task did not affect the results. The descriptive and inferential statistics are presented in the Supplementary Materials (Table S6). 

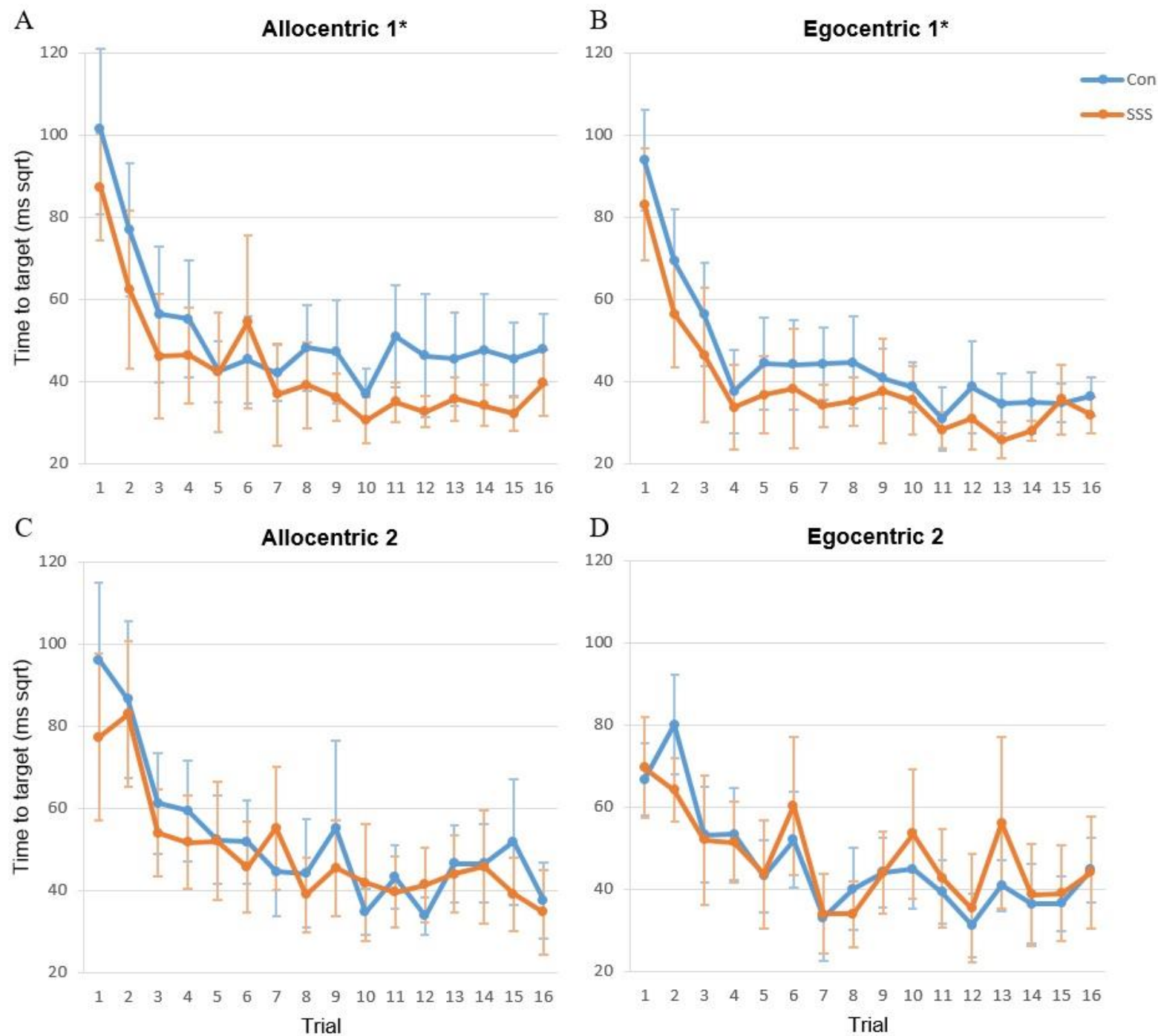

Fig. 5. Graphs showing the performance over trials measured by the time needed to find the platform in the Allocentric 1 and Egocentric 1 conditions ( $A$ and $B$ ) and the Allocentric 2 and Egocentric 2 conditions (C and D). The orange lines show the performance of sequence-space synesthetes and the blue lines the performance of control participants. Error bars reflect the 95\% confidence interval. *Synesthetes performed significantly better in the Allocentric 1 and Egocentric 1 conditions compared to controls. The peaks in the learning curves can be explained by the fact that the starting point (red dot) appeared on the same location for every participant in a particular trial. For instance, the starting point in the $7^{\text {th }}$ trial of Egocentric 2 was already close to the location of the platform, resulting in generally shorter times to find the platform. Likewise, the starting point in the $6^{\text {th }}$ trial of Egocentric 2 was relatively far away from the platform, resulting in generally longer times to find the platform.

\subsubsection{Effects of synesthetic features on performance}

Individual differences in the manifestation of SSS were taken into account in further exploratory analyses to see whether some specific synesthetic features were associated with the enhanced performance of synesthetes. The features taken into account were: association vs. projection, perceiving the spatial form in a two-dimensional vs. three-dimensional space, the ability to mentally rotate the form (yes/no), the ability to mentally move over the form (yes/no), 
the ability to zoom in and out (yes/no), whether the form itself moves with time (yes/no) and the presence of additional visual features like colour (yes/no). A synesthete was classified as having the ability if he or she demonstrated or reported this ability for at least one of the sequences. These features were taken as between-subject factors in separate repeated measures ANOVAs with only the synesthete group. None of the features predicted performance reliably, possibly due to the small sizes of the synesthesia sub-groups; but a few trends can be inspected in the Supplementary Materials (concerning the ability to mentally move over the form and whether the form itself moves with time; Fig. S5-S7). Figure 6 concerns our specific hypothesis of enhanced performance of synesthetes with the ability to mentally rotate their spatial forms as opposed to synesthetes without this ability in the Allocentric 1 condition. The figure shows a slightly faster learning curve for synesthetes with this ability, but the analysis did not reach significance (first eight trials, $F(1,21)=2.72, p=.11, \eta_{\mathrm{p}}{ }^{2}=.12$ ), possibly due to the small subsample sizes (synesthetes with the rotation ability $\mathrm{N}=15$, synesthetes without this ability $\mathrm{N}=$ 8).

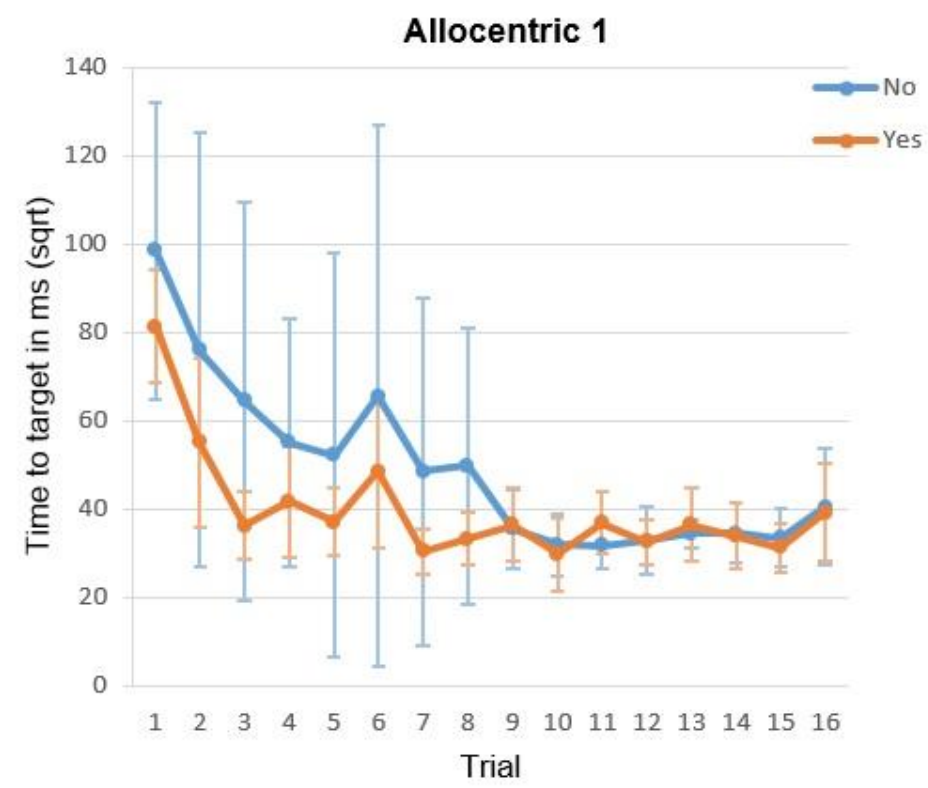

Fig. 6. Graph showing the performance over trials measured by the time needed to find the platform in the Allocentric 1 condition. The orange line shows the performance of synesthetes with the ability to mentally rotate their spatial forms and the blue line the performance of synesthetes without this ability. Error bars reflect the $95 \%$ confidence interval. Synesthetes with the mental rotation ability seemed to perform slightly better during the learning phase, but the analysis did not reach significance.

Next to these exploratory analyses with synesthetic features, it was examined whether the performance of synesthetes was modulated by the complexity of their synesthetic 
experience. A complexity score - based on synesthetic features and synesthetes' drawings was taken as a covariate in a repeated measures ANCOVA with the within-subjects factor trial (16 trials). This complexity score did not significantly modulate the performance of synesthetes for any condition (all $F(1,21)<2.74$, n.s.).

Furthermore, participants were asked about their daily navigation strategies, but due to insufficient difference in used strategies among participants, this factor could not be taken into analysis. Most participants reported to use allocentric navigation strategies or a combination of both allocentric and egocentric strategies, whereas almost no one reported to favour egocentric navigation strategies.

\section{Discussion}

The aim of this study was to investigate whether sequence-space synesthetes show an advantage in spatial navigation. To test this, sequence-space synesthetes and control participants performed a virtual Morris Water Maze task involving two allocentric and two egocentric navigation conditions in which they had to find a hidden platform. Synesthetes were expected to outperform non-synesthetes because of a possible general spatial advantage due to their enhanced sensitivity to spatial relations. Additionally, synesthetes with the ability to mentally rotate their spatial arrangements were expected to outperform synesthetes without this ability.

Indeed, sequence-space synesthetes showed better performance in one of the allocentric conditions and one of the egocentric conditions (i.e., Allocentric 1 and Egocentric 1, the two original test conditions developed by Feigenbaum and Morris, 2004). This group difference was only reliable for one of the two dependent variables (i.e., the time taken to find the platform and not the length of the path that was taken). Additionally, we should mention here that, when examining the range of the SSS and control scores, not all sequence-space synesthetes performed equally well; there are considerable individual differences among synesthetes and some synesthetes performed worse than any controls. As such, our results should be interpreted with caution.

Synesthetes with the ability to mentally rotate their spatial forms did not perform significantly better compared to synesthetes without this ability, despite showing a slightly enhanced learning curve in the Allocentric 1 condition. This is noteworthy, because as Ring et al. (2018) suggested, especially for this condition, mental rotation is important for successful performance. Participants had to change position while the platform and the objects remained fixed. Participants thus saw the display from a different perspective on every trial and had to 
mentally rotate it back to its original perspective (Ring et al., 2018). For the egocentric condition, in which sequence-space synesthetes showed enhanced performance as well, none of the synesthetic features that we took into account were found to specifically contribute to their better performance. In this task, the platform and the participant kept the same positions, while the objects rotated. We suggest that the observed enhanced performance of synesthetes emerged from a generalised spatial advantage rather than direct usage of their visuospatial forms during the MWM task. This is in line with previous findings of synesthesia studies showing generally improved performance in the domain of the synesthetic concurrent experience, outside of the synesthetic experience itself (e.g., grapheme-colour synesthetes demonstrating enhanced colour processing and mirror-touch synesthetes demonstrating enhanced tactile processing; Yaro \& Ward, 2007; Banissy et al., 2009).

It may seem surprising that sequence-space synesthetes did not perform significantly better than controls in the other allocentric and egocentric tasks (i.e., Allocentric 2 and Egocentric 2, the two conditions added by Ring et al., 2018). However, in this allocentric task, the platform moved along with the objects while the participant kept the same position and in this egocentric task, the platform moved along with the participant while the objects remained fixed. This movement of the platform with either the objects or the participant would never happen in everyday environments (Ring et al., 2018). Normally, when we navigate to certain destinations (e.g., buildings), these buildings do not change in space. They remain at fixed locations, like the platform did in the Allocentric 1 and Egocentric 1 tasks. Therefore, the first allocentric and egocentric tasks seemed to better reflect spatial navigation in daily life. An analysis with all four conditions in one repeated measures ANOVA confirmed that the Allocentric 2 and Egocentric 2 conditions were significantly more difficult than the Allocentric 1 and Egocentric 1 conditions $\left(F(1,42)=4.35, p=.043, \eta_{\mathrm{p}}{ }^{2}=.09\right)$. Averaged over trials, it took more time to find the platform in the second conditions $(M=48.97, S D=15.88)$ compared to the first conditions $(M=44.59, S D=12.83)$. Figure 5 clearly demonstrates this as well by less smooth learning curves for these conditions compared to the Allocentric 1 and Egocentric 1 conditions.

The enhanced performance of synesthetes compared to controls in the two original task conditions cannot be explained by any differences between synesthetes and control participants in the ability to follow the task instructions, since there were no differences between groups in any of the control measures (i.e., the number of times they left the pool area, the number of times they lifted the pen from the screen and the number of times they could not find the platform within 60 seconds). Moreover, the experienced time interval between tasks was not 
different between groups, indicated by the time they needed to complete the distractor task. An alternative explanation for the observed group difference in task performance is that sequencespace synesthetes might have been more interested in participating, causing them to be more motivated to perform well. However, this alternative explanation cannot fully account for the observed results, because there was no performance difference between synesthetes and controls in either the place learning condition (i.e., the control task), the Allocentric 2 and Egocentric 2 conditions or the learning curve over trials. The existence of a motivational difference between groups would have been evident in differences in performance here.

Ring et al. (2018) only found differences between groups in the original task conditions as well. Interestingly, they found that individuals with ASD performed significantly worse than control participants in the Allocentric 1 task, while the current study demonstrated that individuals with SSS performed significantly better than controls in this exact same task. This implies that the previously found link between ASD and synesthesia is not reflected in performance on this virtual MWM navigation task. It is possible that the two groups do not converge to similar performance on the MWM task, given that spatial navigation is a complex mental task involving many sub-processes (e.g., Behrmann \& Shomstein, 2009; Harris et al., 2012). Moreover, recent studies indicate that the shared cognitive characteristics between SSS and ASD seem to mainly involve elevated attention to detail (Mealor et al., 2016; Ward et al., 2017; Van Leeuwen et al., 2019).

An interesting question is whether SSS is an adaptive, rather than an epiphenomenal, cognitive function. Perhaps the synesthetic visuospatial experiences remain to exist throughout generations because they are beneficial for a broad range of cognitive functions, either directly aiding task performance (e.g., visualising months helps remembering important events and dates; Simner et al., 2009; and visualising weeks helps easily keeping an overview of things that need to be done) or enhancing cognition in general (e.g., enriched visual imagery; Havlik et al., 2015; Price, 2009; Rizza \& Price, 2012; and improved working memory; Hale et al., 2014). When sequence-space synesthetes are asked whether they experience any benefits of their visuospatial forms, most of them indeed report to experience a memory advantage and state that they cannot imagine living without the visuospatial forms. Easily remembering important dates, like birthdays, is besides a memory advantage socially relevant as well. SSS thus clearly has personal importance. The current data, demonstrating a navigational benefit, adds to the debate whether SSS could indeed be an adaptive cognitive function.

Concerning the methodological aspects of the current study, there is one important difference compared to the study performed by Ring et al. (2018). We focused in our analyses 
on the time that was needed to find the target and the length of the path that was taken to find the target, while they focused on the percentage of time that was spent in the target quadrant. The latter measure, however, did not seem to be most suitable, in contrast to what Ring et al. (2018) suggested. The first reason to doubt this measure is that during one of the conditions (Allocentric 2) the platform was always at the border of two quadrants (i.e., in between the ball and chair and moved along with these objects). It was therefore not possible to correctly define the target quadrant for trials in this condition. The second reason was the absence of a correct reflection of a learning effect over trials (Fig. S1 and S3 in the Supplementary Materials). We assumed that the time spent in the target quadrant should increase over trials, when participants started spending more time searching in the correct quadrant containing the platform. In practice, we observed that after learning participants moved in one straight line from the starting point to the platform. In this way, they spent almost no time searching in the quadrant containing the platform. Ring et al. (2018) chose this measure because of its frequent use in the literature and its reduced vulnerability to variation among participants since it is expressed as a percentage of the total search time. Ring et al. (2018) suggested that a high variation among participants might have added noise to the data of Time to Target and Path Length and possibly obscured any differences between groups. However, based on our measurements and observations, the reasons they put forward do not seem to outweigh the two major problems that come along with this measure.

A caveat of the current study is that we did not include a measure of imagery to check for possible differences in imagery skills between the synesthete and control group. Previous reports suggest enhanced imagery in SSS, and imagery has a beneficial role in spatial navigation. If we had matched the groups on their imagery skill, we could have ruled out imagery as a potentially confounding factor in our study and the enhanced performance of synesthetes might have been more easily attributed to the presence of SSS. Similarly, an additional mental rotation task would have been valuable in confirming whether mental rotation performance was indeed correlated to performance in the Allocentric 1 condition. The slightly enhanced performance of synesthetes with the ability to mentally rotate their spatial forms, observed during the learning phase of this allocentric task, was not significant. A positive correlation between a mental rotation task and the Allocentric 1 condition therefore would have added to the evidence in favour of the contribution of synesthetes' mental rotation ability to performance in this condition. Future studies should aim to replicate our observation of faster learning during allocentric navigation with a larger sample size of synesthetes. The current 
study only included fifteen synesthetes with the mental rotation ability and eight synesthetes without this ability.

One might argue that the Allocentric 1 condition could still be egocentric due to continuous updating of participants' own spatial position relative to the platform during their movement in between trials to any of the four sides of the screen. So instead of finding the platform by using the object cues, participants might represent and update the relation between the platform and their own position (Simons \& Wang, 1998). This so-called viewer-centered representation may trigger an egocentric strategy. In order to ensure that the Allocentric 1 condition can only be solved by using allocentric navigation strategies, in future studies, participants could be moved in a different way disrupting visual, vestibular and proprioceptive information. This prevents the updating mechanism to adjust for changes in participants' position (e.g., participants could be moved in a spinning wheelchair while covering their eyes, like in Simons and Wang, 1998). Another suggestion for future studies is to conduct a navigation experiment in a virtual reality set-up. Such a realistic set-up would convey different and perhaps converging information in order to answer the question whether sequence-space synesthetes have enhanced spatial navigation skills. A virtual reality experiment could thus expand on the current findings.

In addition to the results of the MWM task, this study gave new insights into reliable classification of sequence-space synesthetes and control participants. The consistency test (Rothen et al., 2016) was a valuable addition to check whether synesthetes consistently chose the same locations for items of sequences, but using only a consistency cut-off score would have led to an incorrect classification of participants. For the average area-based consistency score, Rothen et al. (2016) suggested to use a cut-off score of $0.2029 \%$ of the total monitor area, resulting in a cut-off score of 4207 for our study. Using this score would have led to the classification presented in Figure 7, which is highly deviant from the classification based on self-report and drawings. Rothen et al. (2016), however, increased the fit of their classification by excluding participants who had used certain strategies (e.g., placing items of a sequence on a horizontal straight line). We did not exclude these control participants which could explain this classification difference. Rothen et al. (2016) excluded these participants based on visual inspection. Recently, an automated approach for detecting strategies used by non-synesthetes has been developed (Ward, Ipser, Phanvanova, Brown, Bunte \& Simner, 2018). This approach determines the variability of the $\mathrm{x}$ - and/or $\mathrm{y}$-coordinates of the placement of items. Participants with insufficient variability (e.g., using the same location for every item) are automatically reassigned to the non-SSS group. This automated approach is a step forward in improving the 
reliability of the consistency test, but this specific approach will still leave some strategies undetected. For example, non-synesthetes arranging items of a sequence in several rows or columns will reach, besides a high consistency score, sufficient variability and will thus be wrongly allocated to the SSS group.

\begin{tabular}{|c|c|c|c|}
\hline & & \multicolumn{2}{|c|}{ Consistency test } \\
\hline & & SSS & Con \\
\hline \multirow{2}{*}{ 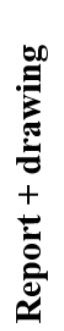 } & के & 20 & 3 \\
\hline & 己ี & 13 & 8 \\
\hline
\end{tabular}

Fig. 7. Scheme representing the suggested classification of participants based on the consistency test (Rothen et al., 2016) and the actual classification based on self-report and drawings. Three sequence-space synesthetes and thirteen control participants would have been misclassified when using the proposed consistency cut-off score.

Instead of only using the results of the consistency test, drawings and participants' descriptions of their synesthetic experiences gave more confidence in correctly distinguishing sequence-space synesthetes from controls. Most studies investigating synesthesia have distinguished synesthetes from controls by using both subjective reports and consistency tests (Brang, Teuscher, Ramachandran, \& Coulson, 2010). The current study demonstrated that drawings of spatial forms can serve as an important classification tool as well. Drawings of synesthetes were generally more complex and characterized by certain shapes (e.g., closed forms), while controls commonly arranged items of a sequence in rows or columns. This is consistent with previous reports of synesthetes experiencing months mostly in circular arrangements, while controls use rows or single straight lines as a default (e.g., Brang et al., 2010; Eagleman, 2009). Importantly, synesthetes showed a tendency to connect items of a sequence within a form by blocks or lines. None of the control participants did this. This fits with synesthetes' reports that an item of a sequence - in particular, months and days - often "encompasses a region of space rather than a single location" (Brang et al., 2010, p. 316). Next to shape and complexity, this tendency of connection is therefore an important feature that characterizes synesthetes' drawings and may contribute to classification. 


\section{Conclusion}

Sequence-space synesthetes have enhanced spatial navigation skills in a virtual navigation task. This study demonstrates that synesthetes are faster in locating a target, but not more efficient in the path taken to the target, and only in tasks that are most similar to real life situations. This study provides the first potential evidence for a spatial navigation benefit in SSS and the next question is whether these results translate to spatial navigation in daily environments. The results contribute to the growing body of literature concerning the cognitive benefits of SSS and suggest that enhanced spatial navigation skills could emerge from generally enhanced visuospatial abilities in sequence-space synesthetes. The association of SSS with enhanced spatial navigation implicates an important evolutionary advantage of having SSS.

\section{Acknowledgements}

We would like to thank Dr. Melanie Ring for providing the MWM task and Dr. Nicolas Rothen for providing the consistency task. We thank the Technical Support Group (Radboud University Nijmegen, Faculty of Social Sciences) for their invaluable help with making the MWM task run on the touchscreen and arranging the task set-up. We are grateful to all our participants for their interest and time investment and we would like to thank Dr. Surya Gayet and the reviewers for valuable comments and suggestions for improvement of the manuscript.

\section{Funding}

This work was supported by a Netherlands Organisation for Scientific Research Veni grant (451.14.025) to TvL.

\section{Competing interests}

The authors declare no competing interests.

\section{Data and materials accessibility statement}

The anonymised responses to the questionnaire, the raw data files of the MWM task and consistency task, as well as Matlab code and the final data files used for analysis are made available in a digital repository, accessible through the following link: http://hdl.handle.net/11633/aacpshmf. Task code for the MWM task can be retrieved from: https://osf.io/dvfwb/ (Ring, M., Gaigg, S., Barr, P., \& Bowler, D. M. (2019, September 27). Testing allocentric versus egocentric spatial navigation using the watermaze task in humans). Task code for the consistency test and Matlab code for generating visualisations of the spatial 
forms can be retrieved from: https://osf.io/6hq94/ (Rothen, N., Ward, J., Jünemann, K., Mealor, A. D., \& Burckhardt, V. (2019, October 1). The sensitivity and specificity of a diagnostic test of sequence-space synesthesia).

Pre-registration

No part of the study procedures or analyses was pre-registered prior to the research being undertaken. 


\section{References}

Banissy, M. J., Walsh, V., \& Ward, J. (2009). Enhanced sensory perception in synaesthesia. Experimental Brain Research, 196 (4), 565-571. doi:10.1007/s00221-009-1888-0

Behrmann, M., \& Shomstein, S. (2009). Spatial cognition and executive function. Encyclopedia of Neuroscience, 173-179. doi:10.1016/B978-008045046-9.00423-X

Bohobot, V. D., Lerch, J., Thorndycraft, B., Iaria, G., \& Zijdenbos, A. P. (2007). Gray matter differences correlate with spontaneous strategies in a human virtual navigation task. Journal of Neuroscience, 27(38), 10078-10083. doi:10.1523/JNEUROSCI.176307.2007

Brang, D., Miller, L. E., McQuire, M., Ramachandran, V. S., \& Coulson, S. (2013). Enhanced mental rotation ability in time-space synaesthesia. Cognitive Processing, 14(4), 429434. doi:10.1007/s10339-013-0561-5

Brang, D., Teuscher, U., Ramachandran, V. S., \& Coulson, S. (2010). Temporal sequences, synesthetic mappings, and cultural biases: the geography of time. Consciousness and Cognition, 19(1), 311-320. doi:10.1016/j.concog.2010.01.003

Chersi, F., \& Burgess, N. (2015). The cognitive architecture of spatial navigation: hippocampal and striatal contributions. Neuron, 88(1), 64-77. doi:10.1016/j.neuron.2015.09.021

Cohen Kadosh, R., Gertner, L., \& Terhune, D. B. (2012). Exceptional abilities in the spatial representation of numbers and time: insights from synesthesia. The Neuroscientist, 18(3), 208-215. doi:10.1177/1073858411402835

Di Tore, P. A., Corona, F., \& Sibilio, M. (2014). Orienteering: spatial navigation strategies and cognitive processes. Journal of Human Sport \& Exercise, 10(1). doi:10.14198/jhse.2015.10.Proc1.45

Eagleman, D. M. (2009). The objectification of overlearned sequences: A new view of spatial sequence synaesthesia. Cortex, 45(10), 1266-1277. doi:10.1016/j.cortex.2009.06.012

Faul, F., Erdfelder, E., Lang, A.-G., \& Buchner, A. (2007). G*Power 3: A flexible statistical power analysis program for the social, behavioral, and biomedical sciences. Behavior Research Methods. 39(2), 175-191. doi:10.3758/bf03193146

Feigenbaum, J. D., \& Morris, R. G. (2004). Allocentric versus egocentric spatial memory after unilateral temporal lobectomy in humans. Neuropsychology, 18(3), 462-472. doi:10.1037/0894-4105.18.3.462 
Gertner, L., Henik, A., \& Cohen Kadosh, R. (2009). When 9 is not on the right: Implications from number-form synesthesia. Consciousness and Cognition, 18(2), 366-374. doi:10.1016/j.concog.2009.02.005

Guariglia, C., Piccardi, L., Iaria, G., Nico, D., \& Pizzamiglio, L. (2005). Representational neglect and navigation in real space. Neuropsychologia, 43(8), 1138-1143. doi:10.1016/j.neuropsychologia.2004.11.021

Hale, J., Thompson, J. M., Morgan, H. M., Cappelletti, M., \& Cohen Kadosh, R. (2014). Better together? The cognitive advantages of synaesthesia for time, numbers, and space. Cognitive Neuropsychology, 31(7-8), 545-564. doi:10.1080/02643294.2014.967759

Harris, M. A., Wiener, J. M., \& Wolbers, T. (2012). Aging specifically impairs switching to an allocentric navigational strategy. Frontiers in Aging Neuroscience, 4, 29. doi:10.3389/fnagi.2012.00029

Havlik, A. M, Carmichael, D. A, \& Simner, J. (2015). Do sequence-space synaesthetes have better spatial imagery skills? Yes, but there are individual differences. Cognitive Processing, 16(3), 245-253. doi:10.1007/s10339-015-0657-1

Hughes, J. E. A., Gruffydd, E., Simner, J., \& Ward, J. (2019). Synaesthetes show advantages in savant skill acquisition: Training calendar calculation in sequence-space synaesthesia. Cortex, 113, 67-82. doi:10.1016/j.cortex.2018.11.023

Iaria, G., Petrides, M., Dagher, A., Pike, B., \& Bohobot, V. D. (2003). Cognitive strategies dependent on the hippocampus and caudate nucleus in human navigation: variability and change with practice. Journal of Neuroscience, 23(13), 5945-5952. doi: 10.1523/JNEUROSCI.23-13-05945.2003

Jonas, C. N., \& Price, M. C. (2014). Not all synesthetes are alike: spatial vs. visual dimensions of sequence-space synaesthesia. Frontiers in Psychology, 5, 1171. doi:10.3389/fpsyg.2014.01171

Konishi, K., \& Bohobot, V. D. (2013). Spatial navigational strategies correlate with gray matter in the hippocampus of healthy older adults tested in a virtual maze. Frontiers in Aging Neuroscience, 5, 1. doi:10.3389/fnagi.2013.00001

Mealor, A. D., Simner, J., Rothen, N., Carmichael, D. A., \& Ward, J. (2016). Different dimensions of cognitive style in typical and atypical cognition: new evidence and a new measurement tool. PLoS ONE, 11(5), e0155483. doi:10.1371/journal.pone.0155483 
Morris, R. G. (1981). Spatial localization does not require the presence of local cues. Learning and Motivation, 12(2), 239-260. doi:10.1016/0023-9690(81)90020-5

Price, M. C. (2009). Spatial forms and mental imagery. Cortex, 45(10), 1229-1245. doi:10.1016/j.cortex.2009.06.013

Price, M. C., \& Pearson, D. G. (2013). Toward a visuospatial developmental account of sequence-space synaesthesia. Frontiers in Human Neuroscience, 7, 689. doi:10.3389/fnhum.2013.0 0689

Ring, M., Gaigg, S. B., Altgassen, M., Barr, P., \& Bowler, D. M. (2018). Allocentric versus egocentric spatial memory in adults with autism spectrum disorder. Journal of Autism and Developmental Disorders, 48(6), 2101-2111. doi:10.1007/s10803-018-3465-5

Rizza, A., \& Price, M. C. (2012). Do sequence-space synaesthetes have better spatial imagery skills? Maybe not. Cognitive Processing, 13, 299-303. doi:10.1007/s10339-012-04597

Rothen, N., Jünemann, K., Mealor, A. D., Burckhardt, V., \& Ward, J. (2016). The sensitivity and specificity of a diagnostic test of sequence-space synaesthesia. Behavior Research Methods, 48(4), 1476-1481. doi:10.3758/s13428-015-0656-2

Rothen, N., Meier, B., \& Ward, J. (2012). Enhanced memory ability: Insights from synaesthesia. Neuroscience and Biobehavioral Reviews, 36(8), 1952-1963. doi:10.1016/j.neubiorev.2012.05.004

Simner, J., Mayo, N., \& Spiller, M. J. (2009). A foundation for savantism? Visuo-spatial synaesthetes present with cognitive benefits. Cortex, 45(10), 1246-1260. doi:10.1016/j.cortex.2009.07.007

Simons, D. J., \& Wang, R. F. (1998). Perceiving real-world viewpoint changes. Psychological Science, 9(4), 315-320. doi:10.1111/1467-9280.00062

Smilek, D., Callejas, A., Dixon, M. J., \& Merikle, P. M. (2007). Ovals of time: Time-space associations in synaesthesia. Consciousness and Cognition, 16(2), 507-519. doi:10.1016/j.concog.2006.06.013

Van Leeuwen, T. M., van Petersen, E., Burghoorn, F., Dingemanse, M. \& van Lier, R. (2019). Autistic traits in synaesthesia: Atypical sensory sensitivity and enhanced perception of details. Philosophical Transactions of the Royal Society B, 374(1787). doi: 10.1098/rstb.2019.0024

Ward, J., Hoadley, C., Hughes, J. E., Smith, P., Allison, C., Baron-Cohen, S., \& Simner, J. (2017). Atypical sensory sensitivity as a shared feature between synaesthesia and autism. Scientific Reports, 7, 41155. doi:10.1038/srep41155 
Ward, J., Ipser, A., Phanvanova, E., Brown, P., Bunte, I., \& Simner, J. (2018). The prevalence and cognitive profile of sequence-space synaesthesia. Consciousness and cognition, 61, 79-93. doi:10.1016/j.concog.2018.03.012

Ward, J., Sagiv, N., \& Butterworth, B. (2009). The impact of visuo-spatial number forms on simple arithmetic. Cortex, 45(10), 1261-1265. doi:10.1016/j.cortex.2009.03.017

Yaro, C., \& Ward, J. (2007). Searching for Shereshevskii: what is superior about the memory of synaesthetes? Quarterly journal of experimental psychology (2006), 60(5), 681-695. doi:10.1080/17470210600785208 